\title{
Estudio ecocardiográfico y de la concentración de NT-proBNP en pacientes diabéticos tipo 2 con y sin cardiopatía isquémica
}

\author{
Sara Castaño Rodrígueza ${ }^{a}$, Isabel Coma-Canella ${ }^{a}$, Begoña López Salazarb y Joaquín Barba Cosialsa
}

aDepartamento de Cardiología. Clínica Universidad de Navarra. Pamplona. Navarra. España.

bÁrea de Ciencias Cardiovasculares. Centro de Investigación Clínica Aplicada (CIMA). Universidad de

Navarra. Pamplona. Navarra. España.

El objetivo de este trabajo es analizar posibles diferencias ecocardiográficas y bioquímicas (NT-proBNP) entre controles y pacientes diabéticos tipo 2 sin cardiopatía isquémica y con ella. El estudio ecocardiográfico comprendió la forma y la función ventricular izquierda. Además se determinaron las concentraciones plasmáticas de NT-proBNP. Los diabéticos sin cardiopatía isquémica presentaron mayor prevalencia de disfunción diastólica (el 88 frente al 74\%; $p<0,001$ ) y mayor concentración de NT-proBNP $(350,6 \pm 197,8$ frente a $281,7 \pm 190,4 \mathrm{fmol} /$ $\mathrm{ml} ; \mathrm{p}<0,001)$ que los controles. Los diabéticos con cardiopatía isquémica mostraron concentraciones de NTproBNP muy superiores a las de los diabéticos sin cardiopatía isquémica $(720,4 \pm 278,1$ frente a $350,6 \pm 197,8$ $\mathrm{fmol} / \mathrm{ml}$ respectivamente; $\mathrm{p}<0,001)$. Una concentración de NT-proBNP > $490 \mathrm{fmol} / \mathrm{ml}$ tuvo el $84 \%$ de sensibilidad y el $75 \%$ de especificidad para detectar cardiopatía isquémica en pacientes diabéticos.

Palabras clave: Miocardiopatía diabética. Cardiopatía isquémica. Disfunción diastólica. BNP.

\section{Echocardiographic Findings and NT-proBNP Level in Type-2 Diabetic Patients With and Without Ischemic Heart Disease}

The aim of this study was to determine whether there are differences in echocardiographic findings or in the level of a biochemical marker (i.e. N-terminal probrain natriuretic peptide [NT-proBNP]) between controls and type-2 diabetic patients with or without ischemic heart disease. Echocardiography was used to assess left ventricular function and morphology. In addition, the plasma NT-proBNP concentration was measured. The prevalence of diastolic dysfunction was greater in diabetics without ischemic heart disease than in controls ( $88 \%$ vs. $74 \%$, respectively; $P<.001$ ) and the NT-proBNP concentration was higher (350.6 \pm 197.8 vs. $281.7 \pm 190.4$ $\mathrm{fmol} / \mathrm{mL} ; P<.001)$. Diabetics with ischemic heart disease had a higher NT-proBNP concentration than those without $(720.4 \pm 278.1$ vs. $350.6 \pm 197.8 \mathrm{fmol} / \mathrm{mL}$, respectively; $P<.001)$. An NT-proBNP concentration $>490 \mathrm{fmol} / \mathrm{mL}$ had a sensitivity of $84 \%$ and a specificity of $75 \%$ for detecting ischemic heart disease in diabetics.

Key words: Diabetic cardiomyopathy. Ischemic heart disease. Diastolic dysfunction. BNP.

Full English text available from: www.revespcardiol.org

\section{INTRODUCCIÓN}

Los cambios miocárdicos originados por la diabetes mellitus (DM) en ausencia de cardiopatía isquémica $(\mathrm{CI})$, hipertensión arterial u otras causas conocidas de cardiopatía se conocen como miocardiopatía diabética. La disfunción diastólica del ventrículo izquierdo (DDVI) se considera la alteración funcional inicial; además se han descrito cambios estructurales como aumento del grosor miocárdico

Correspondencia: Dra. S. Castaño.

Departamento de Cardiología. Clínica Universitaria de Navarra.

Avda. Pío XII, 36. 31008 Pamplona. Navarra. España.

Correo electrónico: scastano@unav.es

Recibido el 3 de noviembre de 2008.

Aceptado para su publicación el 28 de enero de 2009. y de la masa ventricular izquierda. Finalmente, pueden aparecer aumento de los volúmenes ventriculares y anomalías en la contractilidad ${ }^{1}$.

Algunos estudios con reducido número de pacientes indican que la concentración de NT-proBNP se eleva en los pacientes diabéticos tipo 2 con respecto a la población no diabética sólo cuando la DM se acompaña de complicaciones microvasculares y/o macrovasculares ${ }^{2}$. Conociendo la fisiopatología de la miocardiopatía diabética y los estímulos para la síntesis y la secreción de NT-proBNP, cabe pensar que haya alteraciones ecocardiográficas en la miocardiopatía diabética y que este péptido se encuentre elevado en estos pacientes aun cuando la DM no se acompañe de complicaciones vasculares. Este estudio, con mayor número de pacientes que los previos, va dirigido a comprobar esta hipótesis, de modo que los objetivos de este trabajo son dos: 
analizar posibles diferencias ecocardiográficas y en la concentración de NT-proBNP entre un grupo control y otro de diabéticos sin CI y comprobar si el NT-proBNP podría ser marcador diagnóstico de CI en los pacientes diabéticos.

\section{MÉTODOS}

\section{Población de estudio}

Entre marzo de 2004 y agosto de 2005 se incluyó a 284 pacientes mayores de 30 años que acudieron a la consulta de cardiología para prevención primaria o revisión anual por su CI. Los pacientes se dividieron en tres grupos: 99 sin DM ni CI (control, grupo 1), 110 diabéticos tipo 2 sin CI (grupo 2) y 75 diabéticos tipo 2 con CI (grupo 3). En el grupo control se excluyó la DM o cualquier grado de trastorno del metabolismo de los hidratos de carbono mediante la determinación de una curva de glucemia. Para el diagnóstico de DM2 se utilizaron los criterios propuestos por la American Diabetes Association $^{3}$. Se seleccionó a los pacientes de los grupos 2 y 3 en función de antecedente conocido de CI o isquemia documentada en las pruebas de detección siguiendo las recomendaciones vigentes ${ }^{4}$. Se excluyó del estudio a los pacientes con fracción de eyección ventricular izquierda $<45 \%$ y aquellos con cardiopatías de origen conocido diferente del isquémico.

\section{Estudio ecocardiográfico}

Los estudios fueron realizados por el mismo ecocardiografista con un equipo Sonos HP 5500 con sonda de $3 \mathrm{MHz}$, dotado de segundo armónico y software para Doppler tisular. Se llevó a cabo un estudio morfológico del ventrículo izquierdo. La fracción de eyección se estimó según el método de Teichholz y se calculó la fracción de acortamiento y la fracción de acortamiento mesocárdica según la fórmula de Simone ${ }^{5}$. El estudio de la función diastólica comprendió análisis del flujo transmitral, velocidad de propagación y análisis del Doppler tisular del anillo mitral. Los patrones de llenado mitral se establecieron según los criterios de Khouri et $\mathrm{al}^{6}$ y García et $\mathrm{al}^{7}$.

\section{Determinación del NT-proBNP}

La concentración de NT-proBNP en plasma se determinó por un ELISA comercial, utilizando un antisuero específico contra el extremo aminoterminal del proBNP (Biomedica Gruppe, Alemania).

Los cocientes de variación interanalítica e intraanalítica fueron del 4 y el 5\% respectivamente. La sensibilidad fue de $5 \mathrm{fmol} / \mathrm{ml}$ de NT-proBNP.

\section{Análisis estadístico}

El estudio transversal, descriptivo y comparativo entre variables cualitativas y cuantitativas se realizó mediante las pruebas de la $\chi^{2}$ o ANOVA con test post-hoc de Bonferroni. Las variables cuantitativas se han expresado como media \pm desviación estándar y las categóricas, en porcentajes. Consideramos significativo un valor $\mathrm{p}<0,05$. Para determinar la utilidad del NT-proBNP como marcador de isquemia, se hizo un análisis de las curvas ROC. Se utilizó el paquete estadístico SPSS ${ }^{\circledR} 11.0$ (SPSS Inc.).

\section{RESULTADOS}

Las características clínicas de los pacientes quedan reflejadas en la tabla 1 .

\section{Análisis de la forma y la función sistólica del ventrículo izquierdo}

Las diferencias estructurales quedan reflejadas en la tabla 1. La fracción de acortamiento fue significativamente menor en el grupo 3 que en el 1, y no se observaron otras diferencias en cuanto a la función sistólica.

\section{Análisis de la función diastólica}

Ambos grupos de diabéticos presentaron mayor proporción de pacientes con DDVI que el grupo control, sin diferencias entre los dos grupos de diabéticos (grupo 1,74\%; grupo 2, $88 \%$; grupo 3, 90\%; $\mathrm{p}<0,001$ ) (tabla 2). El porcentaje de contribución auricular al llenado ventricular estimado con la detección automática de bordes fue significativamente mayor en el grupo 2 que en el 1 (tabla 1).

\section{Concentración sérica de NT-proBNP}

El valor de NT-proBNP fue en aumento desde el grupo 1 al 3, con diferencias significativas entre todos los grupos: $281,7 \pm 190,4,350,6 \pm 197,8$ y $720,6 \pm 278,1 \mathrm{fmol} / \mathrm{ml}$ respectivamente $(\mathrm{p}<0,001)$ (fig. 1). No hubo correlación entre la concentración de NT-proBNP y los distintos parámetros y estadios de DDVI.

Se hizo un análisis de las curvas ROC para evaluar la utilidad del NT-proBNP como marcador de $\mathrm{CI}$ en pacientes diabéticos tipo 2 . El área bajo la curva fue de 0,864 $\pm 0,029$ ( $\mathrm{p}<0,0001$ ) (fig. 2). Una concentración $>490 \mathrm{fmol} / \mathrm{ml}$ mostró un $84 \%$ de sensibilidad y un $75 \%$ de especificidad para diagnosticar CI, con un RR de 15,1 (intervalo de confianza del 95\%, 7,07-34,2; $\chi^{2}=56,05$; $\mathrm{p}<0,0001)$. 
TABLA 1. Características clínicas y morfológicas y función sistólica del ventrículo izquierdo

\begin{tabular}{|c|c|c|c|c|}
\hline & Controles $(n=99)$ & Diabéticos $\sin \mathrm{Cl}(\mathrm{n}=110)$ & Diabéticos con Cl $(n=75)$ & $\mathbf{p}$ \\
\hline Varones & $57(57)$ & $65(59)$ & $63(84)$ & $<0,001^{*}$ \\
\hline Edad (años) & $63,9 \pm 9,19$ & $65,1 \pm 8,3$ & $64,8 \pm 8,5$ & 0,35 \\
\hline Peso (kg) & $75,6 \pm 12,9$ & $80,2 \pm 17,1$ & $82,8 \pm 14$ & $0,018^{8}$ \\
\hline Talla (cm) & $164 \pm 9$ & $163,8 \pm 10,6$ & $167,2 \pm 7,8$ & 0,11 \\
\hline IMC & $27,5 \pm 3,9$ & $28,9 \pm 5$ & $30,1 \pm 3$ & 0,15 \\
\hline Cintura (cm) & $98,3 \pm 10,7$ & $103,7 \pm 14,2$ & $105,6 \pm 8,7$ & $0,003^{+}$ \\
\hline Cadera (cm) & $108 \pm 10$ & $109,8 \pm 11,5$ & $111 \pm 12$ & 0,39 \\
\hline HTA & $67(68)$ & $81(74)$ & $51(69)$ & 0,54 \\
\hline \multicolumn{5}{|l|}{ Tabaquismo } \\
\hline Sí & $15(15)$ & $14(13)$ & $12(16)$ & 0,07 \\
\hline No & $61(61,6)$ & $69(63,3)$ & $18(24)$ & $<0,001^{\star}$ \\
\hline Ex fumadores & $23(23)$ & $26(24)$ & $44(59)$ & $<0,001^{\star}$ \\
\hline Hipercolesterolemia & $57(57)$ & $80(73)$ & $54(73)$ & $<0,001^{+}$ \\
\hline $\mathrm{AF}$ de $\mathrm{Cl}$ & $14(14)$ & $18(16)$ & $26(35)$ & $<0,001^{\star}$ \\
\hline $\mathrm{HbA}_{1 \mathrm{c}}(\mathrm{mg} / \mathrm{dl})$ & $5,5 \pm 0,4$ & $6,8 \pm 1,3$ & $7 \pm 1,5$ & $<0,001^{+}$ \\
\hline Creatinina $(\mathrm{mg} / \mathrm{dl})$ & $0,9 \pm 0,1$ & $1,03 \pm 0,6$ & $1,08 \pm 0,2$ & 0,49 \\
\hline $\mathrm{SD}(\mathrm{mm})$ & $10,3 \pm 2,5$ & $10,3 \pm 2,1$ & $10,2 \pm 2,1$ & 0,96 \\
\hline PPD (mm) & $10,8 \pm 2,6$ & $10,7 \pm 1,8$ & $10,6 \pm 1,7$ & 0,84 \\
\hline DTD (mm) & $49,6 \pm 5,2$ & $49,3 \pm 5,3$ & $52 \pm 6$ & $0,006^{*}$ \\
\hline DTS (mm) & $31,7 \pm 5$ & $32,1 \pm 6$ & $34,1 \pm 6,9$ & $0,02^{\&}$ \\
\hline VTD (ml) & $119,6 \pm 28,9$ & $117,9 \pm 29,9$ & $132,2 \pm 30,8$ & $0,009^{*}$ \\
\hline VTS (ml) & $42,2 \pm 16$ & $42,9 \pm 16,6$ & $52,4 \pm 17,1$ & $0,001^{*}$ \\
\hline Masa VI (g) & $199,6 \pm 84,3$ & $198,6 \pm 57,7$ & $210,8 \pm 75,8$ & 0,48 \\
\hline IMV $\left(g / m^{2}\right)$ & $107,9 \pm 43,1$ & $108,4 \pm 30,6$ & $113,7 \pm 43,2$ & 0,68 \\
\hline GRP & $0,43 \pm 0,1$ & $0,44 \pm 0,09$ & $0,41 \pm 0,08$ & 0,15 \\
\hline $\mathrm{FE}(\%)$ & $63 \pm 15$ & $62 \pm 10,5$ & $59 \pm 10$ & 0,13 \\
\hline FA $(\%)$ & $36 \pm 6,4$ & $35 \pm 5,9$ & $33 \pm 7,5$ & $0,027^{8}$ \\
\hline FAm (\%) & $0,17 \pm 0,01$ & $0,16 \pm 0,01$ & $0,16 \pm 0,01$ & 0,79 \\
\hline $\mathrm{FE}_{\mathrm{DAB}}(\%)$ & $57 \pm 7,1$ & $57 \pm 7,7$ & $54 \pm 7,2$ & 0,09 \\
\hline $\mathrm{FVTD}_{\mathrm{DAB}}(\mathrm{ml})$ & $63 \pm 17,4$ & $67 \pm 25,1$ & $80 \pm 29,8$ & $0,001^{*}$ \\
\hline $\mathrm{VTS}_{\mathrm{DAB}}(\mathrm{ml})$ & $27 \pm 9,3$ & $30 \pm 14,3$ & $37 \pm 16$ & $<0,001^{\star}$ \\
\hline $\mathrm{FRA}_{\mathrm{DAB}}(\%)$ & 33,1 & 39 & 34,8 & $0,03^{\#}$ \\
\hline
\end{tabular}

AF de Cl: antecedentes familiares de cardiopatía isquémica; Cintura: perímetro a nivel umbilical; Creatinina: creatinina en sangre; DTD: diámetro telediastólico; DTS: diámetro telesistólico; FA: fracción de acortamiento; FAm: fracción de acortamiento mesocárdica; FE: fracción de eyección; $\mathrm{FE}_{\mathrm{DA}}$ : fracción de eyección por detección automática de bordes; $F R A_{D A B}$ : contribución auricular al llenado ventricular por detección automática de bordes; GRP: grosor relativo de pared; HbA 1 : glucohemoglobina; HTA: hipertensión arterial; IMC: índice de masa corporal; IMVI: índice de masa ventricular izquierda; PPD: pared posterior en diástole; SD: septo interventricular en diástole; VTD: volumen telediastólico; $\mathrm{VTD}_{\mathrm{DAB}}$ : volumen telediastólico por detección automática de bordes; VTS: volumen telesistólico; $\mathrm{VTS}_{\mathrm{DAB}}$ : volumen telesistólico por detección automática de bordes.

*: Grupo 3 frente a los otros dos; \&: grupo 3 frente al 1 ; +: grupo 1 frente a los otros dos; \#: grupo 1 frente al 2.

Las variables cuantitativas se expresan como media \pm desviación estándar y los valores de p se obtuvieron mediante el test de ANOVA. Las variables categóricas se expresan en porcentajes y los valores de $\mathrm{p}$ se obtuvieron mediante el test de la $\chi^{2}$.

TABLA 2. Estudio de la función diastólica

\begin{tabular}{|c|c|c|c|c|}
\hline & Controles $(n=99)$ & Diabéticos $\sin \mathrm{Cl}(\mathrm{n}=110)$ & Diabéticos con $\mathrm{Cl}(\mathrm{n}=75)$ & $\mathbf{p}$ \\
\hline THP (ms) & $75,6 \pm 18,8$ & $73,1 \pm 20,2$ & $76,7 \pm 20,1$ & 0,46 \\
\hline IRTV (ms) & $113,8 \pm 29,9$ & $105,5 \pm 24$ & $113,6 \pm 22,9$ & 0,29 \\
\hline $\mathrm{E} / \mathrm{Em}$ & $9,6 \pm 3,8$ & $9,6 \pm 3,1$ & $9,9 \pm 5,2$ & 0,92 \\
\hline $\mathrm{Em}(\mathrm{cm} / \mathrm{s})$ & $8,3 \pm 3,8$ & $8,4 \pm 2,3$ & $8,3 \pm 2,6$ & 0,83 \\
\hline $\mathrm{Vp}(\mathrm{cm} / \mathrm{s})$ & $56 \pm 22,2$ & $5 \pm 26,7$ & $55 \pm 23,1$ & 0,97 \\
\hline $\mathrm{K}_{\mathrm{VI}}$ & $0,08 \pm 0,04$ & $0,09 \pm 0,05$ & $0,08 \pm 0,05$ & 0,43 \\
\hline$\tau$ & $55,7 \pm 12$ & $55,9 \pm 14,1$ & $56,4 \pm 13,9$ & 0,51 \\
\hline $\mathrm{DD}$ & $73(74)$ & $96(88)$ & $68(90)$ & $<0,001^{+}$ \\
\hline $\mathrm{RR}$ & $69(69)$ & $84(76)$ & $53(70)$ & $<0,006^{\#}$ \\
\hline Seudonormal & $1(1)$ & $12(11)$ & $12(16)$ & $<0,001^{+}$ \\
\hline No clasificados & $3(3)$ & 0 & $3(4)$ & \\
\hline
\end{tabular}

DD: disfunción diastólica; E/Em: relación de la onda E del flujo transmitral (eco-Doppler) con la onda E del anillo mitral (Doppler tisular); IRTV: tiempo de relajación isovolumétrica; $K_{v}$ : constante de rigidez del ventrículo izquierdo; RR: relajación retrasada; THP: tiempo de hemipresión; Vp: velocidad de propagación del anillo mitral; $\tau$ : constante del tiempo de relajación isovolumétrica.

+: grupo 1 frente a los otros dos; \#: grupo 1 frente al 2.

Las variables cuantitativas se expresan como media \pm desviación estándar y los valores de $\mathrm{p}$ se obtuvieron mediante el test de ANOVA. Las variables categóricas se expresan en porcentajes y los valores de $p$ se obtuvieron mediante el test de la $\chi^{2}$. 


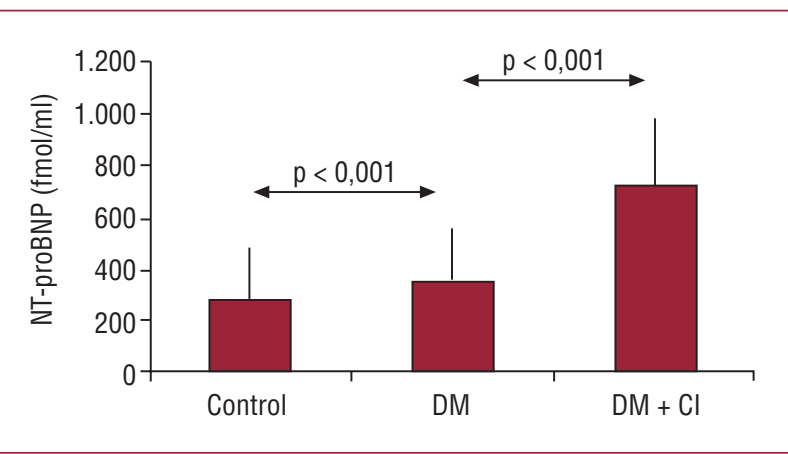

Fig. 1. Concentraciones de NT-proBNP en los tres grupos.

\section{DISCUSIÓN}

En este estudio los diabéticos sin CI mostraron mayores DDVI y concentración de NT-proBNP que los controles de su misma edad. En el grupo de diabéticos con CI, la diferencia fundamental fue la concentración plasmática de NT-proBNP, mucho más alta que en los otros dos grupos. Existen múltiples trabajos que señalan que la DDVI es extremadamente frecuente en diabéticos asintomáticos, y se han detectado anomalías del llenado ventricular izquierdo hasta en un $75 \%$ de los sujetos diabéticos ${ }^{8}$. Los resultados de nuestro estudio se acercan a esa cifra. La media de edad y la prevalencia de hipertensión arterial de los pacientes del grupo control explican la elevada prevalencia de DDVI en este grupo.

Existen estudios en diabéticos en los que la elevación del BNP se correlaciona con hipertrofia ventricular izquierda y disfunción sistólica y diastólica, por lo que constituye un factor pronóstico de morbimortalidad $^{9}$. En este trabajo, a pesar de encontrar más DDVI y concentración de NT-proBNP en los pacientes diabéticos sin CI que en el grupo control, no encontramos correlación entre la elevación del NT-proBNP y los diversos grados de disfunción diastólica.

El péptido natriurético tipo $\mathrm{B}$ y el NT-proBNP tienen elevado valor pronóstico, tanto en la angina estable como en la inestable y el infarto agudo de miocardio ${ }^{10}$. En nuestro estudio, en el grupo de diabéticos con CI se incluyó a pacientes estables y asintomáticos que acudían a la consulta para revisión; en ellos la concentración de NT-proBNP fue muy superior a la de los diabéticos sin CI, lo que indica que este péptido podría ser útil como marcador bioquímico en el diagnóstico de CI en diabéticos, hallazgo interesante que precisa de más estudios para confirmarse. Conviene señalar que la muestra de este estudio representa en buena medida al espectro de pacientes que se pueden beneficiar de la determinación de NT-proBNP, ya que se trata de diabé-

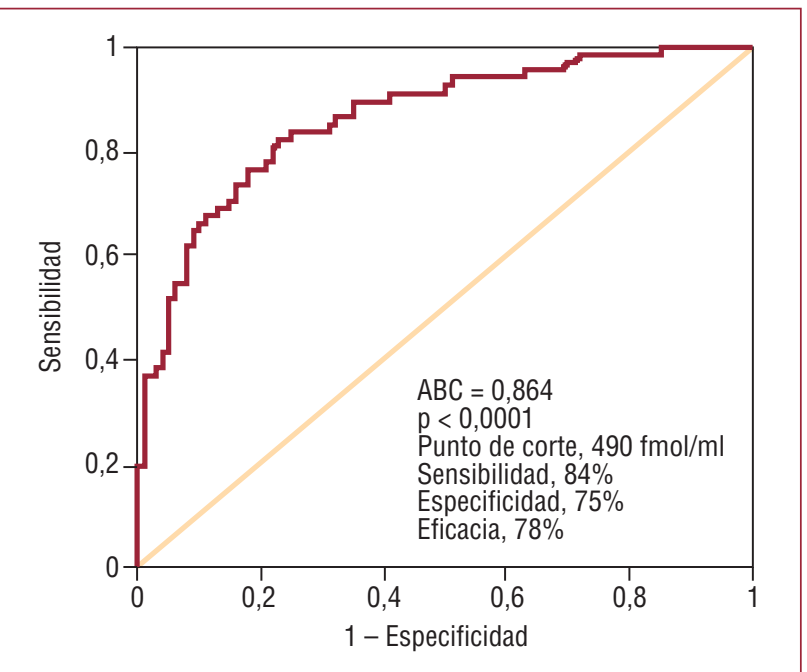

Fig. 2. Curva ROC del NT-proBNP para discriminar el grupo de diabéticos (DM) con y sin cardiopatía isquémica (Cl).

ticos como los que acuden diariamente a las consultas con la finalidad de descartar CI. En ellos la determinación del NT-proBNP con resultados elevados, como los observados en nuestro estudio, podría constituir un marcador de riesgo de CI.

\section{Limitaciones}

Son varios los factores que influyen en la concentración plasmática de NT-proBNP ${ }^{11}$. Entre ellos cabe destacar el sexo y la obesidad, que en nuestro estudio no se distribuyeron por igual en todos los grupos. Teniendo en cuenta que las mujeres presentan valores de NT-proBNP más altos y que la obesidad se asocia a cifras más bajas, ambas variables podrían ocultar diferencias aún más acentuadas entre los grupos, pero en el mismo sentido que las halladas. Otros factores que se distribuyen desigualmente son la hipercolesterolemia y el tabaquismo, pero no se sabe si pueden influir en la concentración de NT-proBNP. Las pruebas de detección de isquemia no tienen sensibilidad del 100\%, por lo que no podemos descartar que algún paciente del grupo de DM sin CI tuviera CI, a pesar de haberse realizado las pruebas de detección cuando estaba indicado. En el grupo de los diabéticos con $\mathrm{CI}$, pese a exigirse una fracción de eyección $>45 \%$ como en los otros dos grupos, no se cuantificó el grado de isquemia. Finalmente, la estimación de la fracción de eyección con el método de Teichholz tiene conocidas limitaciones.

En conclusión, los diabéticos sin CI tienen más DDVI y aumento del NT-proBNP en ausencia de diferencias estructurales y de función sistólica con respecto al grupo control. El NT-proBNP se encuentra elevado en mayor medida en los diabéticos 
con CI. Una concentración de $490 \mathrm{pg} / \mathrm{ml}$ tiene elevadas sensibilidad y especificidad para la detección de $\mathrm{CI}$ en pacientes diabéticos.

\section{BIBLIOGRAFÍA}

1. Struthers AD, Morris AD. Screening for and treating left ventricular abnormalities in diabetes mellitus: a new way of reducing cardiac deaths. Lancet. 2002;359:1430-2.

2. Beer S, Golay S, Bardy D, Feihl F, Gaillard RC, Bachmann $\mathrm{C}$, et al. Increased plasma levels of $\mathrm{N}$-terminal brain natriuretic peptide (NT-proBNP) in type 2 diabetic patients with vascular complications. Diabetes Metab. 2005;31:56773.

3. Diagnosis and Classification of Diabetes Mellitus. Diabetes Care. 2006;29 Suppl. 1:43-8.

4. Consensus Development Conference on the diagnosis of coronary heart disease in people with diabetes: 10-11 February 1998, Miami, Florida. American Diabetes Association. Diabetes Care. 1998;21:1551-9.
5. De Simone G, Devereux R, Roman MJ, Ganau A, Saba PS, Alderman $\mathrm{MH}$, et al. Assesment of left ventricular function by de midwall fraccional shortening/end-systolic stress relation in human hypertension. J Am Coll Cardiol. 1994;23:1444-51.

6. Khouri SJ, Maly GT, Suh DD, Walsh TE. A practical approach to the echocardiographic evaluation of diastolic function. J Am Soc Echocardiogr. 2004;17:290-7.

7. García M, James D, Klein T. New Doppler echocardiographic applications for the study of diastolic function. J Am Coll Cardiol. 1998;32:865-75.

8. Boyer JK, Thanigaraj S, Schechtman KB. Prevalence of ventricular diastolic dysfunction in asymptomatic, normotensive patients with diabetes mellitus. Am J Cardiol. 2004;93:870-5.

9. Bhalla MA, Chiang A, Epshteyn V. Prognostic role of B-type natriuretic peptide levels in patients with type 2 diabetes mellitus. J Am Coll Cardiol. 2004;44:1047-52.

10. Omland T, De Lemos JA. Amino-terminal pro-B-type natriuretic peptides in stable and unestable ischemic heart disease. Am J Cardiol. 2008;101:61-6.

11. De Lemos JA, Hildebrandt P. Amino-terminal pro-B-type natriuretic peptides: testing in general populations. Am J Cardiol. 2008;101:16-20. 